\title{
Equivalence of modified gravity equation to the Clausius relation
}

\author{
Kazuharu Bamba ${ }^{1,}$, Chao-Qiang Geng ${ }^{1,}$, Shin'ichi Nojiri², and Sergei D. Odintsov ${ }^{3, *}$ \\ ${ }^{1}$ Department of Physics, National Tsing Hua University, Hsinchu, Taiwan 300 \\ ${ }^{2}$ Department of Physics, Nagoya University, Nagoya 464-8602, Japan \\ ${ }^{3}$ Instituciò Catalana de Recerca i Estudis Avançats (ICREA) and Institut de Ciencies de l'Espai (IEEC-CSIC), \\ Campus UAB, Facultat de Ciencies, Torre C5-Par-2a pl, E-08193 Bellaterra (Barcelona), Spain
}

\begin{abstract}
We explicitly show that the equations of motion for modified gravity theories of $F(R)$-gravity, the scalar-Gauss-Bonnet gravity, $F(\mathcal{G})$-gravity and the non-local gravity are equivalent to the Clausius relation in thermodynamics. In addition, we discuss the relation between the expression of the entropy and the contribution from the modified gravity as well as the matter to the definition of the energy flux (heat).
\end{abstract}

PACS numbers: 04.50.Kd, 04.70.Dy, 95.36.+x, 98.80.-k

The discovery of black hole (BH) entropy by Bekenstein [1] and the first law of BH thermodynamics [2] with a Hawking temperature [3] implies the fundamental connection between gravitation and thermodynamics. Jacobson has shown that the Einstein equation can be derived from the Clausius relation, $\delta S=\delta Q / T$, in thermodynamics [4]. Here, $S$ is the entropy, $Q$ is the heat, and $T$ is the temperature. In his formulation, $\delta Q$ is interpreted as the energy flux through the local Rindler horizon $\mathcal{H}$ at a free-falling local observer $p_{0}$ :

$$
\delta Q=\int_{\mathcal{H}} T_{\mu \nu} \chi^{\mu} d \Sigma^{\nu}
$$

and $T$ could be the Unruh temperature [5]:

$$
T=\frac{k}{2 \pi}
$$

In Eq. (11), $\chi^{\mu}$ is an approximate local boost Killing field future directed to the past of $p_{0}, T_{\mu \nu}$ is the contribution to the energy-momentum tensor from all ordinary matters, and the integration is over a pencil of generators of $\mathcal{H}$ at $p_{0}$, and $d \Sigma^{\mu}$ is given by $d \Sigma^{\mu}=K^{\mu} d \lambda d A$, where $d A$ is the cross section area element of $\mathcal{H}$ and $K^{\mu}$ is an approximate Killing field generating boost at $p_{0}$ and vanishing at $p_{0}$, which is taken as the future pointing to the inside past of $p_{0}$. In Eq. (2), $k$ is the acceleration of the Killing orbit on which the norm of $\chi^{\mu}$ is unity if $K$ is a tangent vector to the generators of $\mathcal{H}$ with an affine parameter $\lambda\left(\lambda=0\right.$ at $\left.p_{0}\right)$. The formulation was extended to $F(R)$-gravity [6, 7] (for a review of $F(R)$ and other modified gravity theories, see [8]), and to the more general extended gravity theory [9] (see also related discussions in [10]). The first generalization of the relation between the gravitational field equations and thermodynamics to Lanczos-Lovelock gravity has been executed in [11, 12] (see also a recent related work in [13]). Moreover, the entropy functional approach for Lanczos-Lovelock gravity

*Also at Tomsk State Pedagogical University. has extensively been discussed in [14]. This entropy functional approach is the same (except for a four-divergence) as the one used in [9, 15] and its connection with diffeomorphism invariance has been noted in [16], in which it has been stated that in principle, all diffeomorphism invariant theories have an entropic derivation, provided one is willing to accept a particular expression as entropy.

In [7] and [9], the definition of the entropy $S$ was used as [17]:

$$
S=-\frac{2}{T} \oint_{\partial \mathcal{H}} S^{\mu \rho \nu \sigma} \hat{\epsilon}_{\mu \rho} \epsilon_{\nu \sigma}
$$

with

$$
S^{\mu \rho \nu \sigma} \equiv \frac{1}{\sqrt{-g}} \frac{\delta I}{\delta R_{\mu \rho \nu \sigma}}
$$

where $I$ is the action, the integration in Eq. (3) is over the surface enclosing the volume $\mathcal{H}, \epsilon^{\mu \nu}$ is a 2-dimensional volume form, and $\hat{\epsilon}^{\mu \nu}$ is given by $\hat{\epsilon}^{\mu \nu}=\nabla^{\mu} \tilde{\chi}^{\nu}=\epsilon^{\mu \nu} / \bar{\epsilon}$ with $\tilde{\chi}^{\nu}=\chi^{\nu} / k$ and the area element $\bar{\epsilon}$ of the cross section of the horizon. Consequently, in [9] the following formula has been obtained: ${ }^{1}$

$$
T^{\sigma \nu}=2\left[-2 \nabla_{\mu} \nabla_{\rho} S^{\mu \sigma \nu \rho}+S^{\mu \rho \tau \sigma} R_{\mu \rho \tau}^{\nu}\right]+g^{\sigma \nu} \Phi .
$$

Here, $\Phi$ is determined by the conservation law or Bianchi identity. As a result, the equivalence between the equations of motion and the fundamental thermodynamic relation for the generalized theories of gravity has been demonstrated in [9].

\footnotetext{
1 The sign of the first term in the right-hand side (r.h.s.) is different from that in [9], which comes from the difference of the definition of the Riemann tensor. In the present paper, we define the curvatures as,

$$
\begin{gathered}
R=g^{\mu \nu} R_{\mu \nu}, \quad R_{\mu \nu}=R_{\mu \lambda \nu}^{\lambda}, \\
R_{\mu \rho \nu}^{\lambda}=-\Gamma_{\mu \rho, \nu}^{\lambda}+\Gamma_{\mu \nu, \rho}^{\lambda}-\Gamma_{\mu \rho}^{\eta} \Gamma_{\nu \eta}^{\lambda}+\Gamma_{\mu \nu}^{\eta} \Gamma_{\rho \eta}^{\lambda} .
\end{gathered}
$$
}


In the present paper, we study modified gravity theories, in particular (i) $F(R)$-gravity ${ }^{2}$, (ii) the scalar-GaussBonnet gravity inspired by (super)string theories, (iii) $F(\mathcal{G})$-gravity [19] and (iv) the non-local gravity, where $\mathcal{G} \equiv R^{2}-4 R_{\mu \nu} R^{\mu \nu}+R_{\mu \nu \rho \sigma} R^{\mu \nu \rho \sigma}$ is the Gauss-Bonnet invariant $\left(R, R_{\mu \nu}\right.$ and $R_{\mu \nu \rho \sigma}$ are the scalar curvature, the Ricci tensor and the Riemann tensor, respectively) and $F(\mathcal{G})$ is an appropriate function in terms of $\mathcal{G}$, and explicitly show that the equations of motion for these theories are equivalent to the Clausius relation in thermodynamics by applying the formula in Eq. (5). Extended (or modified) gravity theories are frequently studied in the context of effective gravity theories of string theories and supergravity. In addition, these theories have the capability to explain the current accelerated expansion of the universe alternative to the $\Lambda \mathrm{CDM}$ cosmology as well as inflation in the early universe.

In Eq. (11), the energy flux $\delta Q$ is expressed by using the energy-momentum tensor, $T_{\mu \nu}$, of all ordinary matters. The entropy $S$ is defined by Eq. (3) with Eq. (4) for a gravity theory. $\delta Q$ is related to $S$ through the Clausius relation $\delta S=\delta Q / T$. By applying Eqs. (1) and (3) to the Clausius relation, Eq. (5) can be derived. Hence, Eq. (5) corresponds to the equation of motion for the gravity theory, which relates the matter and gravity. For the above four extended gravity theories, we derive the explicit expressions of $\nabla_{\mu} \nabla_{\rho} S^{\mu \sigma \nu \rho}, S^{\mu \rho \tau \sigma} R_{\mu \rho \tau}^{\nu}$ and $\Phi$, which are components on the r.h.s. of Eq. (5), to clearly illustrate the equations of motion from the Clausius relation in thermodynamics. Our investigation is the application of the Jacobson's proposal to derive the Einstein equation as a thermodynamic equation of state in general relativity to modified gravity theories. We use units of $k_{\mathrm{B}}=c=\hbar=1$ and denote the gravitational constant $8 \pi G$ by $\kappa^{2} \equiv 8 \pi / M_{\mathrm{Pl}}^{2}$ with the Planck mass of $M_{\mathrm{Pl}}=G^{-1 / 2}=1.2 \times 10^{19} \mathrm{GeV}$.

A general form of the action describing modified gravity theories (in the below context) is given by

$$
I=\int d^{4} x \sqrt{-g}\left[\frac{\mathcal{F}(R, \phi, X, \mathcal{G})}{2 \kappa^{2}}+\mathcal{L}_{\text {matter }}\right]
$$

where $g$ is the determinant of the metric tensor $g_{\mu \nu}$, $\mathcal{L}_{\text {matter }}$ is the matter Lagrangian, $\phi$ is a scalar field, $X \equiv-(1 / 2) g^{\mu \nu} \nabla_{\mu} \phi \nabla_{\nu} \phi$ is a kinetic term of $\phi\left(\nabla_{\mu}\right.$ is the covariant derivative operator associated with $\left.g_{\mu \nu}\right)$, and $\mathcal{F}(R, \phi, X, \mathcal{G})$ is an arbitrary function in terms of $R$, $\phi, X$ and $\mathcal{G}$. The scalar field $\phi$ is a gravitational partner, e.g., a dilaton, in the case of string theories.

From the action in Eq. (6), the gravitational field equa-

\footnotetext{
2 The equations of motion for $F(R)$-gravity in [6] were derived by using nonequilibrium thermodynamics, while in [7] they were examined in equilibrium thermodynamics with the idea of "localboost-invariance" [18]. In the present paper, however, we apply the generalization of the Jacobson's derivation proposed in [9] to $F(R)$-gravity.
}

tion and the equation of motion for $\phi$ are derived as

$$
\begin{aligned}
& \mathcal{F}_{, R}\left(R_{\mu \nu}-\frac{1}{2} R g_{\mu \nu}\right)=\kappa^{2} T_{\mu \nu}^{(\text {matter })}+\frac{1}{2} g_{\mu \nu}\left(\mathcal{F}-\mathcal{F}_{, R} R\right) \\
& +\nabla_{\mu} \nabla_{\nu} \mathcal{F}_{, R}-g_{\mu \nu} \square \mathcal{F}_{, R}+\frac{1}{2} \mathcal{F}_{, X} \partial_{\mu} \phi \partial_{\nu} \phi \\
& +\left(-2 R R_{\mu \nu}+4 R_{\mu \rho} R_{\nu}{ }^{\rho}-2 R_{\mu}{ }^{\rho \sigma \tau} R_{\nu \rho \sigma \tau}\right. \\
& \left.+4 g^{\alpha \rho} g^{\beta \sigma} R_{\mu \alpha \nu \beta} R_{\rho \sigma}\right) \mathcal{F}_{, \mathcal{G}}+2\left(\nabla_{\mu} \nabla_{\nu} \mathcal{F}_{, \mathcal{G}}\right) R \\
& -2 g_{\mu \nu}\left(\square \mathcal{F}_{, \mathcal{G}}\right) R+4\left(\square \mathcal{F}_{, \mathcal{G}}\right) R_{\mu \nu}-4\left(\nabla_{\rho} \nabla_{\mu} \mathcal{F}_{, \mathcal{G}}\right) R_{\nu}{ }^{\rho} \\
& -4\left(\nabla_{\rho} \nabla_{\nu} \mathcal{F}_{, \mathcal{G}}\right) R_{\mu}{ }^{\rho}+4 g_{\mu \nu}\left(\nabla_{\rho} \nabla_{\sigma} \mathcal{F}_{, \mathcal{G}}\right) R^{\rho \sigma} \\
& -4\left(\nabla_{\rho} \nabla_{\sigma} \mathcal{F}_{, \mathcal{G}}\right) g^{\alpha \rho} g^{\beta \sigma} R_{\mu \alpha \nu \beta}, \\
& \mathcal{F}_{, \phi}+\frac{1}{\sqrt{-g}} \partial_{\mu}\left(\mathcal{F}_{, X} \sqrt{-g} g^{\mu \nu} \partial_{\nu} \phi\right)=0
\end{aligned}
$$

where we have used the following expressions:

$$
\begin{aligned}
& \mathcal{F}_{, R}=\frac{\partial \mathcal{F}(R, \phi, X, \mathcal{G})}{\partial R}, \quad \mathcal{F}_{, X}=\frac{\partial \mathcal{F}(R, \phi, X, \mathcal{G})}{\partial X}, \\
& \mathcal{F}_{, \mathcal{G}}=\frac{\partial \mathcal{F}(R, \phi, X, \mathcal{G})}{\partial \mathcal{G}}, \quad \mathcal{F}_{, \phi}=\frac{\partial \mathcal{F}(R, \phi, X, \mathcal{G})}{\partial \phi}
\end{aligned}
$$

Here, $\square \equiv g^{\mu \nu} \nabla_{\mu} \nabla_{\nu}$ is the covariant d'Alembertian for a scalar field and $T_{\mu \nu}^{\text {(matter) }}$ is the contribution to the energymomentum tensor from all ordinary matters.

Hereafter, we investigate four explicit examples of modified gravity theories.

\section{(i) $F(R)$-gravity}

From the action

$$
I=\int d^{4} x \sqrt{-g}\left[F(R)+\mathcal{L}_{\text {matter }}\right]
$$

we obtain

$$
S^{\mu \nu \rho \sigma}=\frac{F^{\prime}(R)}{2}\left(g^{\mu \nu} g^{\rho \sigma}-g^{\mu \sigma} g^{\nu \rho}\right),
$$

and

$$
\begin{aligned}
\nabla_{\mu} \nabla_{\sigma} S^{\mu \rho \nu \sigma} & =\frac{1}{2}\left(\nabla^{\nu} \nabla^{\rho}-g^{\nu \rho} \square\right) F^{\prime}(R), \\
S^{\mu \rho \tau \sigma} R_{\mu \rho \tau}{ }^{\nu} & =R^{\sigma \nu} F^{\prime}(R) .
\end{aligned}
$$

Here and in what follows, the prime denotes differentiation with respect to the argument of the function $F$ as $F^{\prime}(R)=d F(R) / d R$. On the other hand, the equation in $F(R)$-gravity, corresponding to the Einstein equation, is given by

$$
\begin{aligned}
0= & \frac{1}{2} g_{\mu \nu} F(R)-R_{\mu \nu} F^{\prime}(R)+\nabla_{\mu} \nabla_{\nu} F^{\prime}(R) \\
& -g_{\mu \nu} \square F^{\prime}(R)+\frac{1}{2} T_{\mu \nu}^{(\text {matter })} .
\end{aligned}
$$

By comparing Eq. (14) with Eq. (5) and using Eqs. (12) and (13), we find

$$
\begin{aligned}
T_{\mu \nu} & =T_{\mu \nu}^{\text {(matter) }}, \\
\Phi & =-F(R) .
\end{aligned}
$$




\section{(ii) Scalar-Gauss-Bonnet gravity}

The action is given by

$$
\begin{gathered}
I=\int d^{4} x \sqrt{-g}\left[\frac{R}{2 \kappa^{2}}-\frac{\gamma}{2} g^{\mu \nu} \partial_{\mu} \phi \partial_{\nu} \phi-V(\phi)+f(\phi) \mathcal{G}\right. \\
\left.+\mathcal{L}_{\text {matter }}\right]
\end{gathered}
$$

where $\gamma= \pm 1$. If $\phi$ is a canonical scalar field, $\gamma=1$. On the other hand, if the GB invariant is not included, $\phi$ behaves as a phantom scalar field only when $\gamma=-1$. Moreover, $V(\phi)$ is the potential and $f(\phi)$ is an appropriate function of $\phi$. For this theory, we find

$$
\begin{aligned}
S^{\mu \rho \nu \sigma}= & \frac{1}{4 \kappa^{2}}\left(g^{\mu \nu} g^{\rho \sigma}-g^{\mu \sigma} g^{\nu \rho}\right) \\
& +f(\phi)\left\{\left(g^{\mu \nu} g^{\rho \sigma}-g^{\mu \sigma} g^{\nu \rho}\right) R\right. \\
& -2\left(g^{\rho \sigma} R^{\mu \nu}-g^{\rho \nu} R^{\mu \sigma}-g^{\mu \sigma} R^{\rho \nu}+g^{\mu \nu} R^{\rho \sigma}\right) \\
& \left.+2 R^{\mu \rho \nu \sigma}\right\},
\end{aligned}
$$

and

$$
\begin{aligned}
& \nabla_{\sigma} S^{\mu \rho \nu \sigma}=\nabla_{\sigma} f(\phi)\left\{\left(g^{\mu \nu} g^{\rho \sigma}-g^{\mu \sigma} g^{\nu \rho}\right) R\right. \\
& \left.\quad-2\left(g^{\rho \sigma} R^{\mu \nu}-g^{\rho \nu} R^{\mu \sigma}-g^{\mu \sigma} R^{\rho \nu}\right)+2 R^{\mu \rho \nu \sigma}\right\} \\
& \quad+f(\phi)\left\{\left(g^{\mu \nu} g^{\rho \sigma}-g^{\mu \sigma} g^{\nu \rho}\right) \nabla_{\sigma} R\right. \\
& \quad-2\left(\nabla^{\rho} R^{\mu \nu}-g^{\rho \nu} \nabla_{\sigma} R^{\mu \sigma}-\nabla^{\mu} R^{\rho \nu}+g^{\mu \nu} \nabla_{\sigma} R^{\rho \sigma}\right) \\
& \left.\quad+2 \nabla_{\sigma} R^{\mu \rho \nu \sigma}\right\} .
\end{aligned}
$$

From the Bianchi identity:

$$
0=\nabla_{\mu} R_{\nu \rho \sigma \tau}+\nabla_{\nu} R_{\rho \mu \sigma \tau}+\nabla_{\rho} R_{\mu \nu \sigma \tau},
$$

we have several identities:

$$
\nabla^{\rho} R_{\rho \tau \mu \nu}=\nabla_{\mu} R_{\nu \tau}-\nabla_{\nu} R_{\mu \tau}, \quad \nabla^{\rho} R_{\rho \mu}=\frac{1}{2} \nabla_{\mu} R .
$$

We see that the terms multiplied by $f(\phi)$ (without $\nabla_{\sigma}$ ) cancel with each other and we get

$$
\begin{aligned}
& \nabla_{\sigma} S^{\mu \rho \nu \sigma}=\nabla_{\sigma} f(\phi)\left\{\left(g^{\mu \nu} g^{\rho \sigma}-g^{\mu \sigma} g^{\nu \rho}\right) R\right. \\
& \left.\quad-2\left(g^{\rho \sigma} R^{\mu \nu}-g^{\rho \nu} R^{\mu \sigma}-g^{\mu \sigma} R^{\rho \nu}\right)+2 R^{\mu \rho \nu \sigma}\right\} .
\end{aligned}
$$

Similarly, we find

$$
\begin{array}{r}
\nabla_{\mu} \nabla_{\sigma} S^{\mu \rho \nu \sigma}=\nabla_{\mu} \nabla_{\sigma} f(\phi)\left\{\left(g^{\mu \nu} g^{\rho \sigma}-g^{\mu \sigma} g^{\nu \rho}\right) R\right. \\
\left.\quad-2\left(g^{\rho \sigma} R^{\mu \nu}-g^{\rho \nu} R^{\mu \sigma}-g^{\mu \sigma} R^{\rho \nu}\right)+2 R^{\mu \rho \nu \sigma}\right\},
\end{array}
$$

and

$$
\begin{aligned}
S^{\mu \rho \nu \sigma} R_{\mu \rho \nu}^{\eta}= & \frac{1}{2 \kappa^{2}} R^{\sigma \eta}+f(\phi)\left\{2 R^{\sigma \eta} R-4 R_{\mu \nu}^{\sigma}{ }^{\eta} R^{\mu \nu}\right. \\
& \left.-4 R_{\mu}^{\eta} R^{\mu \sigma}+2 R_{\mu \rho \nu}^{\eta} R^{\mu \rho \nu \sigma}\right\} .
\end{aligned}
$$

In four dimensions, we have the following non-trivial identity:

$$
\begin{aligned}
0= & \frac{1}{2} g_{\mu \nu} \mathcal{G}-2 R R_{\mu \nu}+4 R_{\mu \rho} R_{\nu}{ }^{\rho}-2 R_{\mu}{ }^{\rho \sigma \tau} R_{\nu \rho \sigma \tau} \\
& +4 g^{\alpha \rho} g^{\beta \sigma} R_{\mu \alpha \nu \beta} R_{\rho \sigma} .
\end{aligned}
$$

We can rewrite Eq. (24) as

$$
S^{\mu \rho \nu \sigma} R_{\mu \rho \nu}^{\eta}=\frac{1}{2 \kappa^{2}} R^{\sigma \eta}+\frac{f(\phi) \mathcal{G}}{2} g^{\sigma \eta} .
$$

Now by comparing Eq. (5) with the equation in the scalar-Gauss-Bonnet gravity corresponding to the Einstein equation:

$$
\begin{aligned}
& T_{\mu \nu}^{(\text {matter })}=\frac{1}{\kappa^{2}}\left(R_{\mu \nu}-\frac{1}{2} R g_{\mu \nu}\right) \\
& -\gamma\left(\partial_{\mu} \phi \partial_{\nu} \phi-\frac{1}{2} g_{\mu \nu} \partial_{\rho} \phi \partial^{\rho} \phi\right)+g_{\mu \nu} V(\phi) \\
& -4\left(\nabla_{\mu} \nabla_{\nu} f(\phi)\right) R+4 g_{\mu \nu}(\square f(\phi)) R-8(\square f(\phi)) R_{\mu \nu} \\
& +8\left(\nabla_{\rho} \nabla_{\mu} f(\phi)\right) R_{\nu}{ }^{\rho}+8\left(\nabla_{\rho} \nabla_{\nu} f(\phi)\right) R_{\mu}{ }^{\rho} \\
& -8 g_{\mu \nu}\left(\nabla_{\rho} \nabla_{\sigma} f(\phi)\right) R^{\rho \sigma} \\
& +8\left(\nabla_{\rho} \nabla_{\sigma} f(\phi)\right) g^{\alpha \rho} g^{\beta \sigma} R_{\mu \alpha \nu \beta},
\end{aligned}
$$

we get

$$
\begin{aligned}
T_{\mu \nu}= & T_{\mu \nu}^{(\text {matter })}+\gamma\left(\partial_{\mu} \phi \partial_{\nu} \phi-\frac{1}{2} g_{\mu \nu} \partial_{\rho} \phi \partial^{\rho} \phi\right) \\
& -g_{\mu \nu} V(\phi), \\
\Phi= & \frac{R}{2 \kappa^{2}}-f(\phi) \mathcal{G} .
\end{aligned}
$$

\section{(iii) $F(\mathcal{G})$-gravity}

In the so-called $F(\mathcal{G})$-gravity [19], the action is given by

$$
I=\int d^{4} x \sqrt{-g}\left[\frac{R}{2 \kappa^{2}}+F(\mathcal{G})+\mathcal{L}_{\text {matter }}\right] .
$$

In this case, we find

$$
\begin{aligned}
S^{\mu \rho \nu \sigma}= & \frac{1}{4 \kappa^{2}}\left(g^{\mu \nu} g^{\rho \sigma}-g^{\mu \sigma} g^{\nu \rho}\right) \\
& +F^{\prime}(\mathcal{G})\left\{\left(g^{\mu \nu} g^{\rho \sigma}-g^{\mu \sigma} g^{\nu \rho}\right) R\right. \\
& -2\left(g^{\rho \sigma} R^{\mu \nu}-g^{\rho \nu} R^{\mu \sigma}-g^{\mu \sigma} R^{\rho \nu}+g^{\mu \nu} R^{\rho \sigma}\right) \\
& \left.+2 R^{\mu \rho \nu \sigma}\right\},
\end{aligned}
$$

where $F^{\prime}(\mathcal{G})=d F(\mathcal{G}) / d \mathcal{G}$. By repeating the calculations similar to Eqs. (19)-(26), we obtain

$$
\begin{aligned}
& \nabla_{\mu} \nabla_{\sigma} S^{\mu \rho \nu \sigma}=\nabla_{\mu} \nabla_{\sigma} F^{\prime}(\mathcal{G})\left\{\left(g^{\mu \nu} g^{\rho \sigma}-g^{\mu \sigma} g^{\nu \rho}\right) R\right. \\
& \left.\quad-2\left(g^{\rho \sigma} R^{\mu \nu}-g^{\rho \nu} R^{\mu \sigma}-g^{\mu \sigma} R^{\rho \nu}\right)+2 R^{\mu \rho \nu \sigma}\right\} \\
& S^{\mu \rho \nu \sigma} R_{\mu \rho \nu}^{\eta}=\frac{1}{2 \kappa^{2}} R^{\sigma \eta}+\frac{F^{\prime}(\mathcal{G}) \mathcal{G}}{2} g^{\sigma \eta}
\end{aligned}
$$

The equation of motion corresponding to the Einstein equation is given by

$$
\begin{aligned}
& T_{\mu \nu}^{\text {(matter) }}=\frac{1}{\kappa^{2}}\left(R_{\mu \nu}-\frac{1}{2} R g_{\mu \nu}\right)-g_{\mu \nu}\left(F(\mathcal{G})-\mathcal{G} F^{\prime}(\mathcal{G})\right) \\
& \quad-4\left(\nabla_{\mu} \nabla_{\nu} F^{\prime}(\mathcal{G})\right) R+4 g_{\mu \nu}\left(\square F^{\prime}(\mathcal{G})\right) R \\
& \quad-8\left(\square F^{\prime}(\mathcal{G})\right) R_{\mu \nu}+8\left(\nabla_{\rho} \nabla_{\mu} F^{\prime}(\mathcal{G})\right) R_{\nu}{ }^{\rho} \\
& \quad+8\left(\nabla_{\rho} \nabla_{\nu} F^{\prime}(\mathcal{G})\right) R_{\mu}{ }^{\rho}-8 g_{\mu \nu}\left(\nabla_{\rho} \nabla_{\sigma} F^{\prime}(\mathcal{G})\right) R^{\rho \sigma} \\
& \quad+8\left(\nabla_{\rho} \nabla_{\sigma} F^{\prime}(\mathcal{G})\right) g^{\alpha \rho} g^{\beta \sigma} R_{\mu \alpha \nu \beta} .
\end{aligned}
$$


Table I: Explicit expressions of $\nabla_{\mu} \nabla_{\rho} S^{\mu \sigma \nu \rho}, S^{\mu \rho \tau \sigma} R_{\mu \rho \tau}^{\nu}$ and $\Phi$ in Eq. (5) for four modified gravity theories: (i) $F(R)$-gravity, (ii) the scalar-Gauss-Bonnet gravity, (iii) $F(\mathcal{G})$-gravity and (iv) the non-local gravity.

\begin{tabular}{llll}
\hline \hline Theory & $\nabla_{\mu} \nabla_{\rho} S^{\mu \sigma \nu \rho}$ & $S^{\mu \rho \tau \sigma} R_{\mu \rho \tau}$ & $\Phi$ \\
\hline (i) $F(R)$-gravity & $\frac{1}{2}\left(\nabla^{\nu} \nabla^{\sigma}-g^{\nu \sigma} \square\right) F^{\prime}(R)$ & $R^{\sigma \nu} F^{\prime}(R)$ & $-F(R)$ \\
(ii) Scalar-Gauss-Bonnet gravity & $\nabla_{\mu} \nabla_{\rho} f(\phi)\left\{\left(g^{\mu \nu} g^{\sigma \rho}-g^{\mu \rho} g^{\nu \sigma}\right) R\right.$ & $\frac{1}{2 \kappa^{2}} R^{\sigma \nu}+\frac{f(\phi) \mathcal{G}}{2} g^{\sigma \nu}$ & $\frac{R}{2 \kappa^{2}}-f(\phi) \mathcal{G}$ \\
& $-2\left(g^{\sigma \rho} R^{\mu \nu}-g^{\sigma \nu} R^{\mu \rho}-g^{\mu \rho} R^{\sigma \nu}\right)$ & \\
& $\left.+2 R^{\mu \sigma \nu \rho}\right\}$ & & \\
& $\nabla_{\mu} \nabla_{\rho} F^{\prime}(\mathcal{G})\left\{\left(g^{\mu \nu} g^{\sigma \rho}-g^{\mu \rho} g^{\nu \sigma}\right) R \frac{1}{2 \kappa^{2}} R^{\sigma \nu}+\frac{F^{\prime}(\mathcal{G}) \mathcal{G}}{2} g^{\sigma \nu}\right.$ & $\frac{R}{2 \kappa^{2}}-F(\mathcal{G})$ \\
(iii) $F(\mathcal{G})$-gravity & $-2\left(g^{\sigma \rho} R^{\mu \nu}-g^{\sigma \nu} R^{\mu \rho}-g^{\mu \rho} R^{\sigma \nu}\right)$ & \\
& $\left.+2 R^{\mu \sigma \nu}\right\}$ & \\
(iv) Non-local gravity & $\frac{1}{4 \kappa^{2}}\left(\nabla^{\nu} \nabla^{\sigma}-g^{\nu \sigma} \square\right)(\tilde{f}(\varphi)-\xi)$ & $\frac{1}{2 \kappa^{2}}(1+\tilde{f}(\varphi)-\xi) R^{\sigma \nu} \frac{R}{2 \kappa^{2}}(1+\tilde{f}(\varphi)-\xi)$ \\
\hline \hline
\end{tabular}

By using Eqs. (32) and (33) and comparing Eq. (5) with Eq. (34), we find

$$
\begin{aligned}
T_{\mu \nu} & =T_{\mu \nu}^{(\text {matter })}, \\
\Phi & =\frac{R}{2 \kappa^{2}}-F(\mathcal{G}) .
\end{aligned}
$$

\section{(iv) Non-local gravity}

We now consider the non-local gravity [20, 21]

$$
I=\int d^{4} x \sqrt{-g}\left\{\frac{1}{2 \kappa^{2}} R\left(1+\tilde{f}\left(\square^{-1} R\right)\right)+\mathcal{L}_{\text {matter }}\right\} .
$$

Here, $\tilde{f}$ is an appropriate function in terms of its argument. The above action can be rewritten by introducing two scalar fields $\varphi$ and $\xi$ in the following form [21]:

$$
\begin{gathered}
I=\int d^{4} x \sqrt{-g}\left[\frac{1}{2 \kappa^{2}}\left\{R(1+\tilde{f}(\varphi))-\partial_{\mu} \xi \partial^{\mu} \varphi-\xi R\right\}\right. \\
\left.+\mathcal{L}_{\text {matter }}\right]
\end{gathered}
$$

which leads to

$$
S^{\mu \rho \nu \sigma}=\frac{1}{4 \kappa^{2}}(1+\tilde{f}(\varphi)-\xi)\left(g^{\mu \nu} g^{\rho \sigma}-g^{\mu \sigma} g^{\nu \rho}\right),
$$

and

$$
\begin{aligned}
\nabla_{\mu} \nabla_{\sigma} S^{\mu \rho \nu \sigma} & =\frac{1}{4 \kappa^{2}}\left(\nabla^{\nu} \nabla^{\rho}-g^{\nu \rho} \square\right)(\tilde{f}(\varphi)-\xi) \\
S^{\mu \rho \nu \sigma} R_{\mu \rho \nu}^{\eta} & =\frac{1}{2 \kappa^{2}}(1+\tilde{f}(\varphi)-\xi) R^{\sigma \eta}
\end{aligned}
$$

The equation of motion corresponding to the Einstein equation is given by

$$
\begin{aligned}
& T_{\mu \nu}^{(\text {matter })}=\frac{1}{\kappa^{2}}\left[-\frac{1}{2} g_{\mu \nu}\left\{R(1+\tilde{f}(\varphi)-\xi)-\partial_{\rho} \xi \partial^{\rho} \varphi\right\}\right. \\
& +R_{\mu \nu}(1+\tilde{f}(\varphi)-\xi)-\frac{1}{2}\left(\partial_{\mu} \xi \partial_{\nu} \varphi+\partial_{\mu} \varphi \partial_{\nu} \xi\right) \\
& \left.+\left(g_{\mu \nu} \square-\nabla_{\mu} \nabla_{\nu}\right)(\tilde{f}(\varphi)-\xi)\right]
\end{aligned}
$$

By using Eqs. (40) and (41) and comparing Eq. (5) with Eq. (42), we find

$$
\begin{aligned}
T_{\mu \nu}= & T_{\mu \nu}^{(\text {matter })}+\frac{1}{2 \kappa^{2}}\left(\partial_{\mu} \xi \partial_{\nu} \varphi+\partial_{\mu} \varphi \partial_{\nu} \xi\right. \\
& \left.-g_{\mu \nu} \partial_{\rho} \xi \partial^{\rho} \varphi\right) \\
\Phi= & \frac{R}{2 \kappa^{2}}(1+\tilde{f}(\varphi)-\xi) .
\end{aligned}
$$

We should note that there is an ambiguity in the separation into $T_{\mu \nu}$ part and $\Phi$ part. For example, instead of Eqs. (43) and (44), we may choose

$$
\begin{aligned}
\tilde{T}_{\mu \nu} & =T_{\mu \nu}^{(\text {matter })}+\frac{1}{2 \kappa^{2}}\left(\partial_{\mu} \xi \partial_{\nu} \varphi+\partial_{\mu} \varphi \partial_{\nu} \xi\right), \\
\tilde{\Phi} & =\frac{R}{2 \kappa^{2}}(1+\tilde{f}(\varphi)-\xi)-\partial_{\rho} \xi \partial^{\rho} \varphi .
\end{aligned}
$$

Here, the last term in Eq. (43) has been included in the definition of $\tilde{\Phi}$.

From the analysis of the above four modified gravity theories, it is clear that we have derived explicit expressions of $\nabla_{\mu} \nabla_{\rho} S^{\mu \sigma \nu \rho}, S^{\mu \rho \tau \sigma} R_{\mu \rho \tau}^{\nu}$ and $\Phi$ in Eq. (5). The results are summarized in Table I. A general expression for $\Phi$ can be expressed as the linear combination of $R /\left(2 \kappa^{2}\right)$, which is the Lagrangian describing general relativity, and the Lagrangian of gravity $\mathcal{L}_{\text {gravity }}$ as

$$
\Phi=c_{1} \frac{R}{2 \kappa^{2}}+c_{2} \mathcal{L}_{\text {gravity }},
$$

where $c_{1}$ and $c_{2}$ are constants. For (i) $F(R)$-gravity, $c_{1}=0, c_{2}=-1$ and $\mathcal{L}_{\text {gravity }}=F(R)$. For (ii) the scalarGauss-Bonnet gravity, $c_{1}=2, c_{2}=-1$ and $\mathcal{L}_{\text {gravity }}=$ $R /\left(2 \kappa^{2}\right)+f(\phi) \mathcal{G}$. For (iii) $F(\mathcal{G})$-gravity, $c_{1}=2, c_{2}=-1$ and $\mathcal{L}_{\text {gravity }}=R /\left(2 \kappa^{2}\right)+F(\mathcal{G})$. For (iv) the non-local gravity, if we use the expression in Eq. (44), we find $c_{1}=0, c_{2}=1$ and $\mathcal{L}_{\text {gravity }}=\left[R /\left(2 \kappa^{2}\right)\right](1+\tilde{f}(\varphi)-\xi)$. If we use Eq. (46) instead of Eq. (44), we obtain $\mathcal{L}_{\text {gravity }}=$ $\left[R /\left(2 \kappa^{2}\right)\right](1+\tilde{f}(\varphi)-\xi)-\partial_{\rho} \xi \partial^{\rho} \varphi$, which is nothing but the gravity part of the action in Eq. (38).

Thus, we have reinforced the generalization [9] in modified gravity theories of the Jacobson's proposal to express 
the Einstein equation as a thermodynamic equation of state in general relativity with our analysis. Our results could support the idea that gravitation on a macroscopic scale is a manifestation of the thermodynamics of the vacuum state of quantum field theory [9].

Between Eqs. (43,44) and Eqs. (45,46), we have find the ambiguity to define $T_{\mu \nu}$. As we see now, this could be a result from the ambiguity when we consider the thermodynamics in the extended gravities. In general, any gravity equation can be written as

$$
T_{\mu \nu}^{(\text {matter })}+T_{\mu \nu}^{\text {(modified gravity) }}=\frac{1}{\kappa^{2}}\left(R_{\mu \nu}-\frac{1}{2} R g_{\mu \nu}\right) .
$$

Hence, if we include the contribution from $T_{\mu \nu}^{\text {(modified gravity) }}$, which comes from the modification of the Einstein gravity, to the definition of the energy flux (heat), the usual area law of the entropy is not modified but the entropy includes the contribution from the (modified) gravity. On the other hand, we may write Eq. (48) as

$$
\begin{aligned}
& T_{\mu \nu}^{\text {(matter) }}=\frac{1}{\kappa^{2}}\left(R_{\mu \nu}-\frac{1}{2} R g_{\mu \nu}\right)+G_{\mu \nu}^{\text {modified gravity }}, \\
& G_{\mu \nu}^{\text {modified gravity }} \equiv-T_{\mu \nu}^{\text {(modified gravity) }} .
\end{aligned}
$$

If we consider the contribution only from matter to the definition of the energy flux (heat), in general the entropy $S$ will be expressed by a function of the area $A$ as $S=$ $h(A)$, where $h(A)$ is an appropriate (not always linear) function in terms of $A$ and it may include the parameters coming from the modified gravity and/or curvatures, etc. Furthermore, there might be a mixture of Eqs. (48) and (49) like

$$
\begin{aligned}
& T_{\mu \nu}^{(\text {matter })}+\tilde{T}_{\mu \nu}^{\text {(modified gravity) }}=\frac{1}{\kappa^{2}}\left(R_{\mu \nu}-\frac{1}{2} R g_{\mu \nu}\right) \\
& +\tilde{G}_{\mu \nu}^{\text {modified gravity }}, \\
& G_{\mu \nu}^{\text {modified gravity }}=-T_{\mu \nu}^{\text {(modified gravity) }} \\
& =\tilde{G}_{\mu \nu}^{\text {modified gravity }}-\tilde{T}_{\mu \nu}^{\text {(modified gravity) }} .
\end{aligned}
$$

Thus, the entropy contains the contribution not only from the matter but from the modified gravity partially, and the expression of the entropy could be modified from the Einstein gravity. This may tell that when we discuss the entropy, we may clarify the contribution to the entropy is purely from the matter or partially from (modified) gravity. Then especially in case that the theory includes the scalar field(s), we cannot always apply Wald's formula in Eq. (33) so naively.

In conclusion, we have explicitly illustrated that the equations of motion for modified gravity theories, in particular $F(R)$-gravity, the scalar-Gauss-Bonnet gravity, $F(\mathcal{G})$-gravity and the non-local gravity, are equivalent to the Clausius relation in thermodynamics. In modified gravity theories, whether we include the contribution from the matter with or without the modified gravity to the definition of the energy flux (heat) is crucial to the expression of the entropy. This point is closely related to the discussion in [22] where it shows that it is possible to obtain a picture of equilibrium thermodynamics on the apparent horizon in the expanding cosmological background for a wide class of modified gravity theories due to a suitable definition of an energy momentum tensor of the component from modified gravity that respects to a local energy conservation.

K.B. and C.Q.G. thank Professor Shinji Tsujikawa for very helpful discussions. K.B. acknowledges the KEK theory exchange program for physicists in Taiwan and the very kind hospitality at KEK and Nagoya University. The work is supported in part by the National Science Council of R.O.C. under Grant \#s: NSC-952112-M-007-059-MY3 and NSC-98-2112-M-007-008-MY3 and National Tsing Hua University under the Boost Program and Grant \#: 97N2309F1 (K.B. and C.Q.G.); MEC (Spain) project FIS2006-02842 and AGAUR (Catalonia) 2009SGR-994, by JSPS Visitor Program (Japan) and by LRSS project N.2553.2008.2 (S.D.O.); and Global COE Program of Nagoya University provided by the Japan Society for the Promotion of Science, G07 (S.N.).
[1] J. D. Bekenstein, Phys. Rev. D 7, 2333 (1973).

[2] J. M. Bardeen, B. Carter and S. W. Hawking, Commun. Math. Phys. 31, 161 (1973).

[3] S. W. Hawking, Commun. Math. Phys. 43, 199 (1975) [Erratum-ibid. 46, 206 (1976)].

[4] T. Jacobson, Phys. Rev. Lett. 75, 1260 (1995).

[5] W. G. Unruh, Phys. Rev. D 14, 870 (1976).

[6] C. Eling, R. Guedens and T. Jacobson, Phys. Rev. Lett. 96, 121301 (2006).

[7] E. Elizalde and P. J. Silva, Phys. Rev. D 78, 061501 (2008).

[8] S. Nojiri and S. D. Odintsov, eConf C0602061, 06 (2006) [Int. J. Geom. Meth. Mod. Phys. 4, 115 (2007)] arXiv:hep-th/0601213].
[9] R. Brustein and M. Hadad, Phys. Rev. Lett. 103, 101301 (2009).

[10] M. K. Parikh and S. Sarkar, arXiv:0903.1176 [hep-th].

[11] T. Padmanabhan, AIP Conf. Proc. 861, 179 (2006) arXiv:astro-ph/0603114.

[12] A. Paranjape, S. Sarkar and T. Padmanabhan, Phys. Rev. D 74, 104015 (2006).

[13] D. Kothawala and T. Padmanabhan, Phys. Rev. D 79, 104020 (2009).

[14] T. Padmanabhan, Gen. Rel. Grav. 40, 529 (2008).

[15] S. F. Wu, G. H. Yang and P. M. Zhang, Prog. Theor. Phys. 120, 615 (2008).

[16] T. Padmanabhan, arXiv:0903.1254 [hep-th].

[17] R. M. Wald, Phys. Rev. D 48, 3427 (1993). 
[18] V. Iyer and R. M. Wald, Phys. Rev. D 50, 846 (1994).

[19] S. Nojiri and S. D. Odintsov, Phys. Lett. B 631, 1 (2005) arXiv:hep-th/0508049].

[20] S. Deser and R. P. Woodard, Phys. Rev. Lett. 99, 111301 (2007).
[21] S. Nojiri and S. D. Odintsov, Phys. Lett. B 659, 821 (2008) arXiv:0708.0924 [hep-th]]

[22] K. Bamba, C. Q. Geng and S. Tsujikawa, arXiv:0909.2159 [gr-qc]. 